\title{
Properties improvement of poly(o-methoxyaniline) based supercapacitors: experimental and theoretical behaviour study of self-doping effect
}

\author{
R. Gonçalves ${ }^{1}$, W. A. Christinelli ${ }^{1}$, A. B. Trench $^{1}$, A. Cuesta $^{2}$, E. C. Pereira ${ }^{1, *}$ \\ ${ }^{1}$ Department of Chemistry, Universidade Federal de São Carlos, Mail Box 676, CEP 13565-905, São Carlos - SP, \\ Brazil \\ ${ }^{2}$ Department of Chemistry, School of Natural and Computing Sciences, University of Aberdeen, Aberdeen AB24 3UE, \\ UK \\ "ernesto@ufscar.br
}

\begin{abstract}
Conducting polymers (CP) are very versatile materials with important properties due to their unique structure, including redox behaviour upon electrochemical polarization. Supercapacitors are among the devices in which CP show a promising performance due to their large specific capacitance. However, the major drawback of these materials is their low durability, which is an inbuilt process caused by ion intercalation, a side effect of the redox process. A viable strategy to increase CP stability is, therefore, the inhibition of the ion intercalation/de-intercalation process. Several strategies have been proposed to minimize this effect, among them self-doping. Self-doping makes the redox process mass-transport independent, decreasing the response time and increasing durability. With this purpose, this work have described the synthesis of layer-by-layer films (LBL) of poly(omethoxyaniline)/poly(3-thiopheneacetic acid) (POMA/PTAA) and contributes to understand how the redox process leads to premature aging, and how self-doping minimizes this problem. The main techniques of characterisation used were electrochemical impedance spectroscopy (EIS) experiments and density functional theory (DFT) calculations. EIS provides important information about the electrochemical behaviour, while DFT calculations allowed to characterize, at the molecular level, the changes in the material structure. The association of both techniques helped to understand how self-doping improves the POMA properties. DFT calculations showed that in the layer-by-layer (LBL) films, the twisting of the polymer chain due to the oxidation is $12 \%$ less while the chain shortening is 5\% compared with films prepared POMA by casting. The EIS data showed the effect of these molecular changes in the structure; the swelling of the LBL films is $50 \%$ less than cast ones. These observations are important because the PTAA layer acts beyond a chargecompensator, working as a backbone structure that mechanically stabilize the POMA layer, leading to an increase in the durability, and improving the electrochemical properties.
\end{abstract}

KEYWORDS: Supercapacitor, Self-Doping, Transmission Lines, DFT, Conducting Polymer. 


\section{INTRODUCTION}

The advancement of science in recent decades led to the fast technological development in several segments. Very important among these areas, are the renewable energy sources and energy storage devices, which may offer solutions to the worldwide energy problem. New alternative materials for these applications have been developed, aiming to reduce the energetic and environmental issues [1,2]. Supercapacitors play an important role in targeting these problems. Conducting Polymers (CPs) are very promising materials for application in energy storage devices due to their unique characteristics, such as: high conductivity in the oxidized state, fast doping/undoping process, low cost and low environmental impact [3]. Energy storage in supercapacitors based on CPs occurs by faradaic processes, providing them with a higher capacity per gram (i.e., higher energy density) than conventional double layer capacitors [4-10]. However, it is still a challenge to obtain CPs with high performance and long cycling life-time[11].

CPs unique properties come from the presence of conjugated double bonds throughout the polymer chains. This kind of structure allows a flow of electrons to be created under specific conditions, namely, upon oxidation of the polymer chains. Besides, this redox transformation is reversible. Charges generated in the polymer film during the redox process need to be neutralised by ions with opposite sign, which requires ion transportation from/to the solution [12]. It is well known that this is the rate-determining step for the redox reaction in CPs. Furthermore, it has been shown that ion intercalation during the redox process causes a volume change in the film, resulting in mechanical stress[13]. This is held responsible for the electrochemical degradation of CPs' performance in electrochemical capacitor applications [1,11]. A possible way to avoid this is to develop a self-doped material. In conducting polymers, self-doping can be achieved by incorporation of chemical species interacting with the polymeric chains (but not necessarily bonded to them) that act as counter-ions during oxidation/reduction process[14]. A different approach involves the use of self-assembled materials[15]. In these kind of materials, one layer is electrochemically active, while the other one has charged functional groups, such as sulfonic or carboxylic acid, to compensate the generated charges in the polymeric chains. In addition, the selfdoping effect in layer-by-layer electrodes leads to important improvements in their properties, such as an increase in the specific capacitances[16], extended life cycle[17] and improved electrochromic properties[18].

Electrochemical impedance spectroscopy (EIS) is a powerful tool for the electrochemical characterization of materials. Using this technique, it is possible to extract information about the kinetics of the redox process and the structure of the electrochemical interface[19-23] in the stationary state. The most common way to analyse EI spectra is to use equivalent circuits, in which 
the circuit elements describe the processes occurring at the electrode-electrolyte interface[24]. This

model works well to describe flat and/or roughened electrodes, but in the case of porous ones as, for example, conducting polymers, another type of interpretation including the porosity description is necessary. In the case of porous materials where the pores are deep enough to be considered as channels in the material, the impedance of the electrolyte in the pores, the impedance of the material itself, and the impedance of the interface between them have to be considered[25,26]. There are two limiting cases for two-media systems: (i) the impedance of the two media are comparable, and (ii) the impedance of one medium is much larger than the other one, which can, hence, be neglected. In this sense, transmission-lines (TL) models stand out among the possible forms of analysing impedance data[27,28]. In these cases, the two branches and the one branch TL models are applied, respectively[29]. In CPs, the two branches to be considered are the ionic transport in the pores and the electron transport in the polymer chains, as well as the ionic charge transport at the interface.

Computational methods are an important tool which can be used to describe, from a molecular point of view, those bulk properties of the material that could be reflect in the interface properties. Density Functional Theory (DFT) is one of the most popular and versatile computational methods [30]. It has wide application in the chemical and material sciences to simulate, analyse and predict properties and behaviours of complex system at the atomic scale. DFT is based on the Thomas-Fermi model[31], that aims to describe electron distribution into small volume elements. The application of this tool to conductive polymers has been proposed in different papers[32-35], and usually it is employed to estimate CPs properties investigated by extrapolating the properties of long-oligomers[33,34]. It is important to stress out that due to the size of the system being studied, the method used is approximate, but the obtained results can be used to support the interpretation of experimental data.

We present here a study of the effect of self-doping in poly(o-methoxyaniline)/poly(3thiophene acetic acid) (POMA/PTAA) layer-by-layer films using EIS and DFT calculations.

\section{EXPERIMENTAL}

\subsection{Chemicals and solutions}

Acetonitrile, lithium perchlorate and perchloric acid used were purchased from SigmaAldrich, and were used as received. Ammonium hydroxide and hydrochloric acid, both in analytical grade and supplied by Vetec, were used for $\mathrm{pH}$ adjustment. All aqueous solutions were prepared with Milli-Q water (resistivity > $18.0 \mathrm{M} \Omega \mathrm{cm}$ ). Polymer powders of POMA and PTAA were prepared following previously described methods [36,37]. A polycation solution was prepared adding $20 \mathrm{mg}$ of POMA and $400 \mu \mathrm{L}$ of acetonitrile to $30 \mathrm{~mL}$ of ultra-pure water, after which the $\mathrm{pH}$ 
was measured and checked to be around 3.0. A separate polyanion solution was prepared by adding

$13.5 \mathrm{mg}$ of PTAA to $25 \mathrm{~mL}$ of water, and the $\mathrm{pH}$ was subsequently adjusted to 8.0 with $\mathrm{NH}_{4} \mathrm{OH}$ and $\mathrm{HCl}$ solutions. Then, the solutions were centrifuged at $9000 \mathrm{rpm}$ and the supernatant powder was removed. The washing solutions was prepared by adjusting the $\mathrm{pH}$ of pure water to the $\mathrm{pH}$ of each polymer solution. Finally, the solutions were used in the film assembly apparatus[38].

\subsection{Polymer films preparation}

Indium tin oxide (ITO, resistivity of $10 \Omega \mathrm{cm}^{-1}$ ) was used as substrate for the deposition of the polymer films. The layer-by-layer assembly method consisted in the deposition of alternated layers of the two polymers. Each layer was obtained by deposition from one of the polymeric solutions for $180 \mathrm{~s}$; followed by a washing for $3 \mathrm{~s}$ with a polymer-free solution of the same $\mathrm{pH}$, and terminated by a drying step under $\mathrm{N}_{2}$ flux for $100 \mathrm{~s}$. Alternating between the polycationic and polyanionic polymer solutions, yielded alternating layers of POMA and PTAA. The combination of one POMA layer and one PTAA layer forms a bilayer. Layer-by-layer (LBL) films composed of 60, 80, 100, 120 bilayers were prepared.

Cast POMA films were obtained by dropping a known amount of POMA solution over the ITO substrate. The film was then dried in a vaccum dissector for $24 \mathrm{~h}$ before characterisation. Cast films of consisting of $60,80,100,120 \mu \mathrm{L}$ of POMA per $\mathrm{cm}^{2}$ were prepared.

The ITO substrate was weighed before and after the LBL polymer assembly or the POMA casting, in order to determine the mass of electroactive material. In the case of the cast POMA films all the deposited mass is electroactive. However, in the case LBL films, just half of the deposited mass was assumed to be electroactive, because only the POMA layer is electroactive in the studied potential window in addition to the consideration of the deposited mass of each polymer is the same in the self-assembling method[15].

\subsection{Electrochemical characterizations procedures}

All experiments were performed using an AUTOLAB 302N potentiostat/galvanostat (Eco Chemie, Netherlands) monitored with NOVA software. A conventional one-compartment/threeelectrode cell was used. The polymer-modified ITO substrates were used as working electrodes, an $\mathrm{Ag} / \mathrm{AgCl}$ electrode in $\mathrm{KCl}\left(3.0 \mathrm{~mol} \mathrm{~L}^{-1}\right)$ was used as reference, and a $\mathrm{Pt}$ wire as counter-electrode. Electrochemical experiments were performed in $0.1 \mathrm{~mol} \mathrm{~L}^{-1} \mathrm{LiClO}_{4}$ in acetonitrile. The cyclic voltammetry $(\mathrm{CV})$ was performed in the potential window from $-0.3 \mathrm{~V}$ up to $0.7 \mathrm{~V}$ at $20 \mathrm{mV} \mathrm{s}^{-1}$. EI spectra were obtained using a FRA32M module in the frequency range between $10 \mathrm{mHz}$ and $10 \mathrm{kHz}$ (10 data points per frequency decade), with a modulation amplitude of $10 \mathrm{mV}_{\mathrm{rms}}$. EI spectra were obtained at DC potentials between $-0.3 \mathrm{~V}$ and $0.7 \mathrm{~V}$ in $0.1 \mathrm{~V}$ steps. The values of the electrical parameters were obtained by fitting the experimental spectra to either the one-branch or 
the two-braches transmission-lines model.

\subsubsection{Transmission lines models}

The main difference between the one-branch and the two-branches TL models lies in the polymer branch (Figure 1). The resistance of the POMA cast films is comparable in magnitude to that of the solution in the pores, and it has to be accounted for using a two-branches TL model. On the other hand, the resistance across LBL films is much lower $\left(<10^{-9} \Omega\right)$ than that of the solution in the pores, and can be disregarded. A one-branch TL model, in which the interface is shortconnected with the working-electrode substrate suffices in this case. We would like to note, however, that an actual short-circuit across the polymer does not exist. In fact, it is the properties of the LBL films that lead to their negligibly small resistance what are the main subject of this research.

The solution branch and the interfacial impedance are equally modelled in both cases. $R_{\text {sol }}$ describes the ohmic drop between the reference electrode and the surface of the working electrode; $R_{\text {pore }}$ corresponds to the resistance of the electrolyte inside the pore, which is related to the pore size and shape; and $R_{\mathrm{ct}}$, is the charge-transfer resistance, associated in the case of conductive polymers to the transport and intercalation of ions. In series with latter resistance, there is the charge-transfer capacitance, $C_{\mathrm{ct}}$. This capacitance is necessary to explain the delay between the intercalation of the ion into the polymer film and its effective arrival to the active site. In parallel to these elements, there is the double-layer capacitance, $\mathrm{C}_{\mathrm{d} l}$. 
Figure 1: Graphical representation of the two-braches (top) and the one-branch (bottom) transmission-lines models used to analyse the EIS data.

\subsection{Computational Methods}

Computational calculations were performed after a relaxation step using semi-empirical methods with the PM7[39] basis set in the MOPAC2016 software[40,41]. Then the geometrical optimizations were carried out at DFT level using the MOLOPT basis set[42] with BLYP functional [43]. All the optimizations were carried out using the CP2K quantum chemistry software [44] and the Maxwell High-Performance Computing Cluster at the University of Aberdeen [45]. The Avogadro software [46] was used for building up and visualising the molecules. The building up of the molecules started from the optimized monomers, followed by increasing the polymers size from dimers to hexadecamers step-by-step, following methodologies described in the literature[21,33,34].

The equilibrium distance between POMA chains was estimated using semi empirical methods with the MOPAC2016 software and the PM7 basis set. For this, the reaction path mode of the software was used, in which, starting from an interchain distance of $10 \AA$, the energy of system was calculated after each step of $1.0 \AA$ in order to build energy-distance curves. Additionally the same was calculated for POMA and PTAA system. The following assumptions were taken in the calculations: (i) the two polymers were assumed to have long-distance order, (ii) both were constructed head-to-tail; and (iii) the thermal energy is enough to bring about low-energy changes in the conformation of the polymeric chains. Calculations were performed for the following 
systems: (i) pure, neutral POMA; (ii) POMA in the presence of PTAA chains; (iii) POMA in the presence of perchlorate ions, (iv) positively charge POMA; and (v) positively charged POMA interacting with $\mathrm{ClO}_{4}{ }^{-}$counter ions and (vi) positive charged POMA with PTAA. All these molecular models are important in understanding the oxidation process and the interaction with PTAA.

\section{RESULTS AND DISCUSSION}

\subsection{Structural characteristics and theoretical model description}

The POMA and PTAA polymers were built by adding monomeric units until the normalised energy converges to a steady state value, which is accepted as that of the model material. This approach is a compromise between computer time duration and property accuracy. Figure $\mathrm{S} 1$ in the Supplementary Information presents a plot of the normalised energy vs. the number of monomer units. Since the difference between the simulated tetramer and octamer is very small, we decided to use the smaller one, together with Periodic Boundary Conditions (PBC)[47,48]. Besides, considering the description of the different oxidation states in polyaniline (and its derivatives), the tetramer model unit was chosen[49] in which it is possible that half of the monomeric units characterize one important state in the material, called emeraldine. Furthermore, we investigated both the POMA polymer system, its oxidized form in the presence of perchlorate ions and, finally, the LBL model, i.e., POMA tetramer in the presence of the same number PTAA monomer units. The repetition units used the calculations are shown in Figure S2 of the Supplementary Information.

The bond order $(\mathrm{BO})$ and bond length $(\mathrm{BL})$ of $\mathrm{C}-\mathrm{C}$ bonds in the aromatic rings of reduced POMA are 1.49 and $1.40 \AA$, respectively, with dihedral angles [35] between aromatic rings of $37.2^{\circ}$. These values show that, in this case, the dihedral angle does not affect the conjugation length in the chains. It is important to stress out, however, that it affects the chain-to-chain distance, which will be discussed in the text below. The C-N BL is $1.46 \AA$, with a BO of 0.988 , and the angle formed by the $\mathrm{C}-\mathrm{N}-\mathrm{C}$ bonds is of $119.6^{\circ}$. We have also computed Mulliken charges[50], which provide an estimation of the partial atomic charges, allowing to determine which atoms concentrate charge and to estimate non-conventional hydrogen bonds[51]. $\mathrm{N}$ and $\mathrm{O}$ atoms accumulate partial negative charges of -0.563 and -0.618 , respectively, as expected from their electronegativity.

Next, we simulate charged POMA chains in which one $e^{-}$per monomer has been removed, which lead to a slight change in the conformation of the chain, as shown in Table 1. The rings lose their aromaticity, containing now two double and four single bonds, i.e., acquiring a quinoid form, as previously proposed for POMA from experimental data[52]. The $\mathrm{C}-\mathrm{N}$ bond length is reduced to $1.36 \AA$ and the BO increased to 1.51. This decrease in the bond length is expected, because it 
becomes resonant between a single and a double bond, as also evidenced by the calculated bond

order. The Mulliken charge of the $\mathrm{O}$ atoms remains almost the same, while that of the $\mathrm{N}$ atoms changes its sign and now amounts to 0.987. This indicates that the positive charge, due to the oxidation, occurs mainly over the $\mathrm{N}$ atoms, as expected[53,54]. Besides, the $\mathrm{C}-\mathrm{N}-\mathrm{C}$ bond angle increases to $136.4^{\circ}$. This last aspect is attributed to the positive charge formation in the $\mathrm{N}$ atom, leading to a decrease in the electron repulsion and, consequently, to an increase in the bond angle. Additionally, the dihedral angle changed from $37.2^{\circ}$ to $49.4^{\circ}$, i.e., the oxidation induced an increase in the torsion between two successive monomeric units. All these parameters are summarized in Table 1, and are in accordance with the literature [53-55].

Table 1: Some structural parameters from the DFT calculations for POMA in reduced and oxidized states.

\begin{tabular}{|c|c|c|}
\hline & $\begin{array}{c}\text { Neutral POMA (reduced } \\
\text { form) }\end{array}$ & $\begin{array}{c}\text { Positively charged POMA } \\
\text { (oxidised form) }\end{array}$ \\
\hline Aromatic $\mathrm{C}-\mathrm{C}$ bond length $/ \AA$ & 1.40 & $1.51 / 1.37$ \\
\hline Aromatic $\mathbf{C}-\mathbf{C}$ bond order & 1.49 & $1.12 / 1.97$ \\
\hline C-N bond length / $\AA$ & 1.46 & 1.36 \\
\hline $\mathrm{C}-\mathrm{N}$ bond order & 0.988 & 1.51 \\
\hline $\mathrm{C}-\mathrm{N}-\mathrm{C}$ angle $/^{\circ}$ & 119.6 & 136.4 \\
\hline Rings dihedral angle $/^{\circ}$ & 37.2 & 49.4 \\
\hline N Mulliken charge & -0.618 & +0.987 \\
\hline O Mulliken charge & -0.563 & -0.568 \\
\hline
\end{tabular}

All these structural parameters are important to understand the changes in the polymer chain due to the oxidation process, which will next be analysed in the light of the EIS data.

\subsection{Electrochemical properties}

Figure 2 shows the cyclic voltammograms (CVs) of cast and LBL polymer films. While the specific current per mass of polymer remains almost constant for cast POMA films, it increases considerably in the case of POMA/PTAA LBL films. We have recently proposed an explanation for this behaviour[16]. Despite the increase of the mass-normalized current, it is still possible to see all the fingerprints of the electrochemical behaviour of POMA in LBL films. 

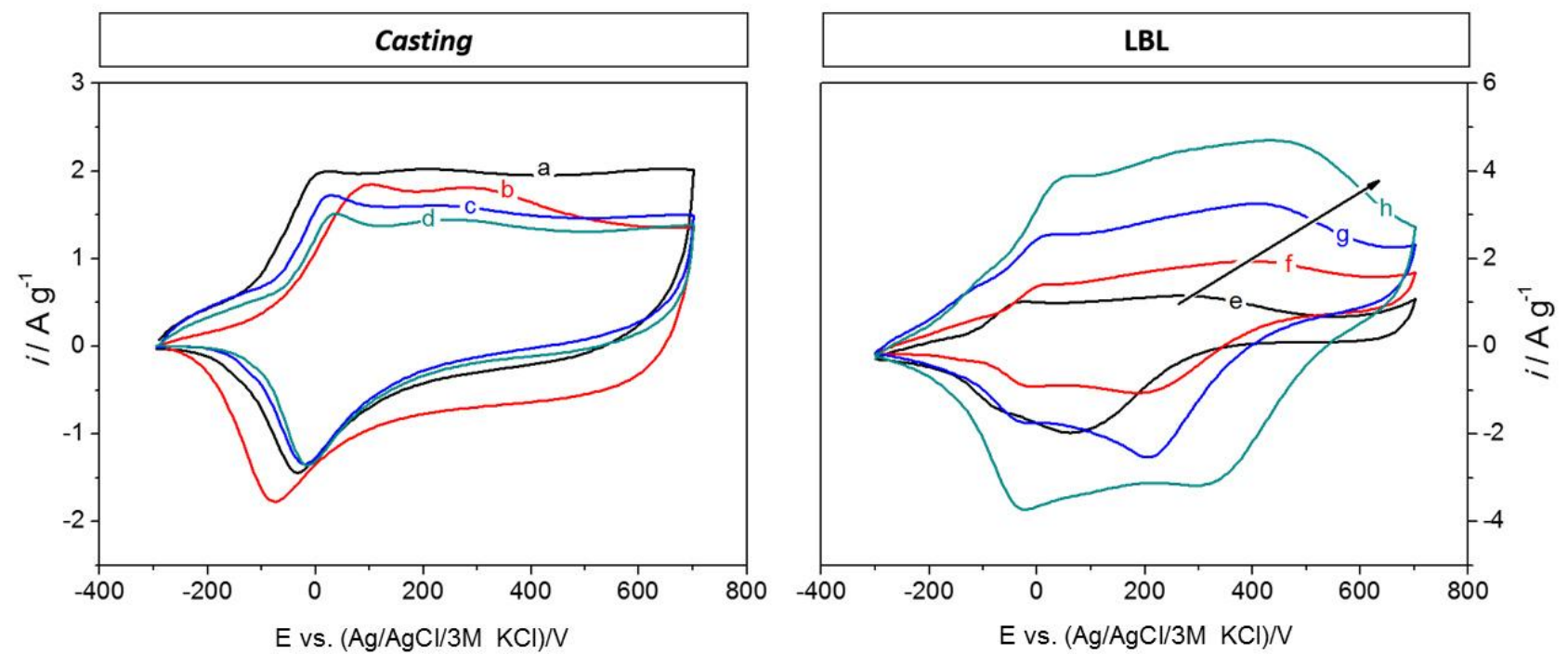

Figure 2: Cyclic voltammograms for cast POMA (left) and LBL POMA/PTAA films (right) in $0.1 \mathrm{~mol} \mathrm{~L}^{-1}$ $\mathrm{LiClO}_{4}$ in actenitrile at $20 \mathrm{mV} \mathrm{s}^{-1}$. (a) 60 (b) 80, (c) 100 and (d) $120 \mu \mathrm{L}$; (e) 60 (f) 80, (g) 100 and (h) 120 bilayers; performed in $\mathrm{LiClO}_{4} 0.1 \mathrm{~mol} \mathrm{~L}^{-1} / \mathrm{ACN}$ at $20 \mathrm{mV} \mathrm{s}^{-1}$.

The models used to analyse the impedance data have been optimized both for the reduced and oxidized states of all the films tested, and allow to describe the changes occurring at different applied potentials. Figure 3 shows typical Bode plots for $80 \mu \mathrm{L}$ POMA cast films and 60 bilayers LBL films in both their reduced and oxidized states. We chose these samples because they present similar mass normalized currents which allow comparable analysis[16]. Clear differences can be observed between the spectra of reduced and oxidised POMA cast films, while no significant difference can be observed in the case of the LBL film. This adds support to the main hypothesis in the corresponding TL impedance model, namely, that the LBL film has a negligible impedance. The oxidation states of POMA are presented in Figure S3 of the Supplementary Information following those description in the literature[56]. As shown in Figure 3, the differences between the oxidised and reduced states are more pronounced at the lower frequency limit, both for cast and LBL films. 

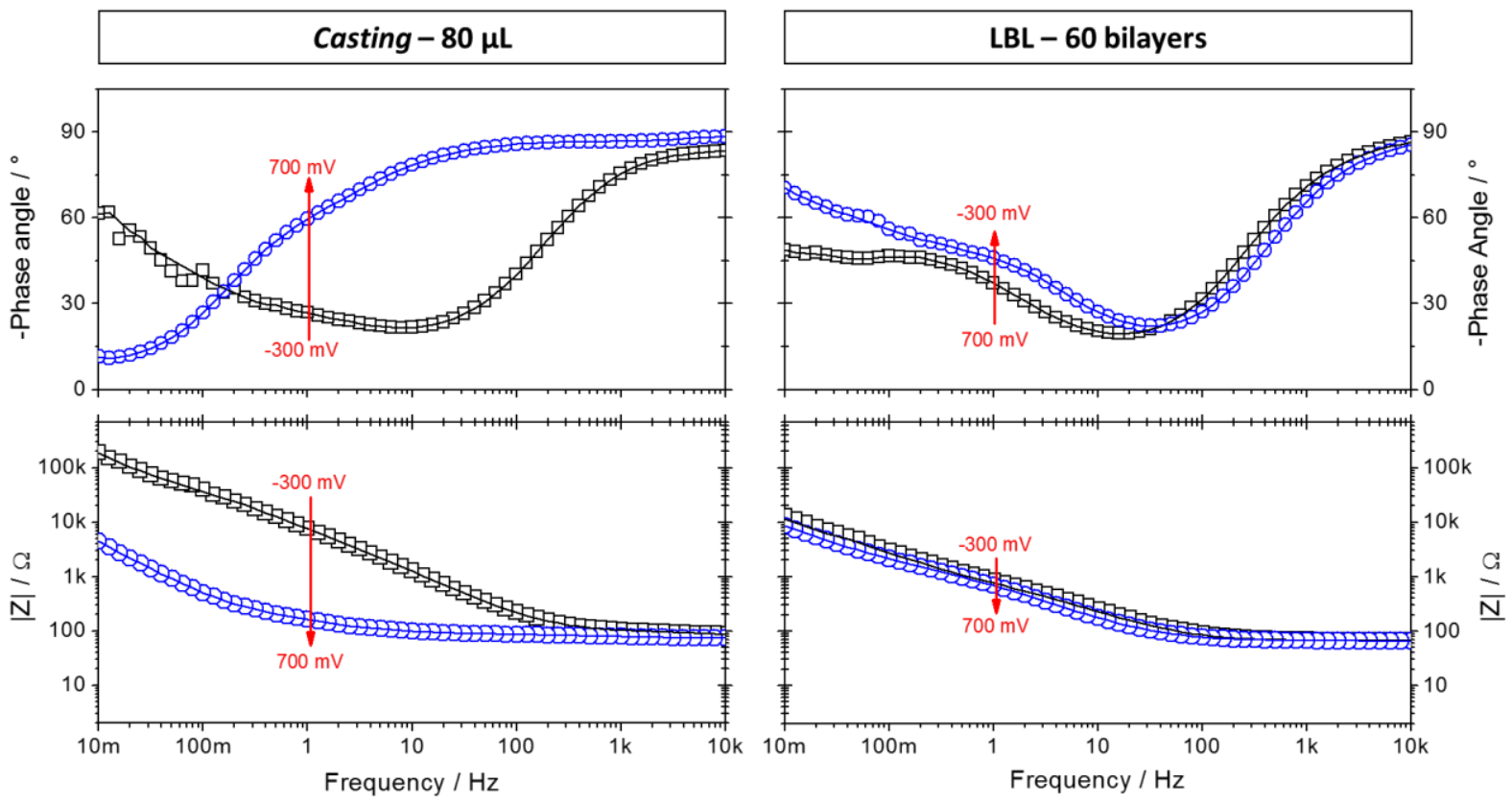

Figure 3: Bode plots for $80 \mathrm{uL}$ POMA cast films (left) and 60 bilayers LBL films (right) in $0.1 \mathrm{~mol} \mathrm{~L}^{-1}$ $\mathrm{LiClO}_{4}$ in acetonitrile at $-0.30 \mathrm{~V}$ (black squares) and $0.70 \mathrm{~V}$ (blue circles). $\Delta V=10 \mathrm{mV}$.

All the parameters describing the behaviour of the polymer films can be obtained from the fit of the experimental impedance spectra to the TL models illustrated in Figure 1. Figure $4 \mathrm{a}$ and $\mathrm{b}$ show the resulting $C_{\mathrm{dl}}$ as a function of the applied potential for all the films prepared. This parameter is associated to the electroactive interface between the polymer and the solution in the pores. Figure $4 \mathrm{c}$ and $4 \mathrm{~d}$ illustrate the relative changes of $C_{\mathrm{dl}}$. Both types of films show an increase in $C_{\mathrm{dl}}$ with increasing potential, as expected from the increase in the volume of the film associated with polymer oxidation [49]. It has been proposed that ion intercalation leads to this volume increase. However, LBL films show an increase of $C_{\mathrm{dl}}$ with potential of ca. $30 \%$ that observed for POMA cast films. This result suggests that the self-doping effect in these materials accounts, at least partially, for charge neutralisation, taking over the role played by ion intercalation, as we have recently suggested [15]. As can also be observed in Figure $4, C_{\mathrm{dl}}$ starts increasing at more negative potentials in the case of the cast films than for LBL ones, which could also be related with the intercalation of ions necessary to compensate the positive charges generated by the oxidation of the POMA chains in the former case. Finally, as can be observed in Figures $4 \mathrm{c}$ and $4 d$, while for LBL films $C_{\mathrm{dl}}$ increases continuously with increasing number of bilayers, for cast films $C_{\mathrm{dl}}$ reaches a maximum for $80 \mu \mathrm{L}$ films, decreasing for thicker POMA films. This can be related to the ohmic drop across the films, which should increase with increasing cast film thickness. As a consequence of this ohmic drop, the oxidation process becomes more difficult and less efficient, and it is not possible to ensure the homogeneity in the oxidation process over the entire film.

Is important to make clear that there is also an increase in the electroactive area, coming 
from the solvated (or at least partially solvated) ions intercalation process during the polymer oxidation[57-59]. It means that, due to the oxidation, there are two factors that contribute to the increase in this capacitance: the increase in the surface area of the polymer and the transformation of the polymer itself from insulate to the its conducting form[60,61]. In this way, we just used the changes in the double-layer capacitance to make qualitative comparisons, since we cannot separate the two involved processes with the experimental approach we used.
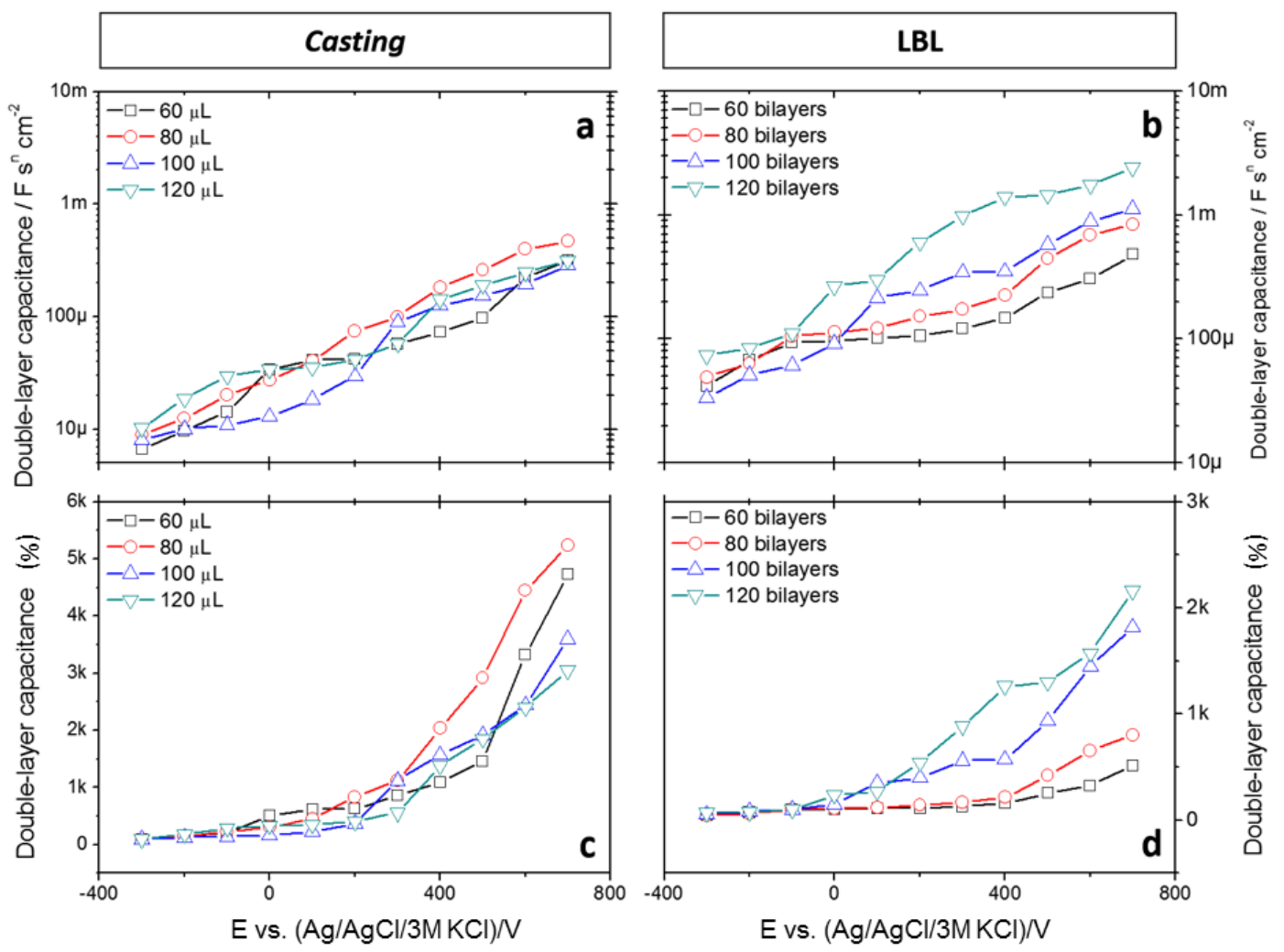

Figure 4: Double-layer capacitance as function of applied potential for casting and LBL films

Figure 5 shows the dependence of $R_{\mathrm{ct}}$ on the applied potential. For the sake of clarity, only one film has been included in Figures $5 \mathrm{a}$ and $5 \mathrm{~b}$, where the corresponding voltammetric response is also shown. $R_{\mathrm{ct}}$ for all the investigated samples are shown in Figures $5 \mathrm{c}$ and $5 \mathrm{~d}$. Cast polymer films show a change in the slope of the decrease of $R_{\mathrm{ct}}$ with potential (Figure 5a), which can be attributed to a change in the electronic conductivity within the polymer film. The current flowing during a voltametric experiment corresponds to the oxidation of the polymer, and the rate of this process is limited by ion intercalation in to the polymer chains in order to neutralise the charges created. When the reduced, low conductive polymer, is transformed to an oxidized, high conductive one, there is a sudden decrease in the ohmic drop of the films, which makes $R_{\mathrm{ct}}$ decrease faster with applied potential. Since the rate of oxidation of LBL films is only partially dependent on the ion 
intercalation process [15], such a change in the slope of the $R_{\mathrm{ct}}$ vs. $E$ plot is not observed (Figure $5 b$ ), except for a large number of bilayers. This could be an indication that there is a maximum number of bilayers where the self-doping mechanism is effective.
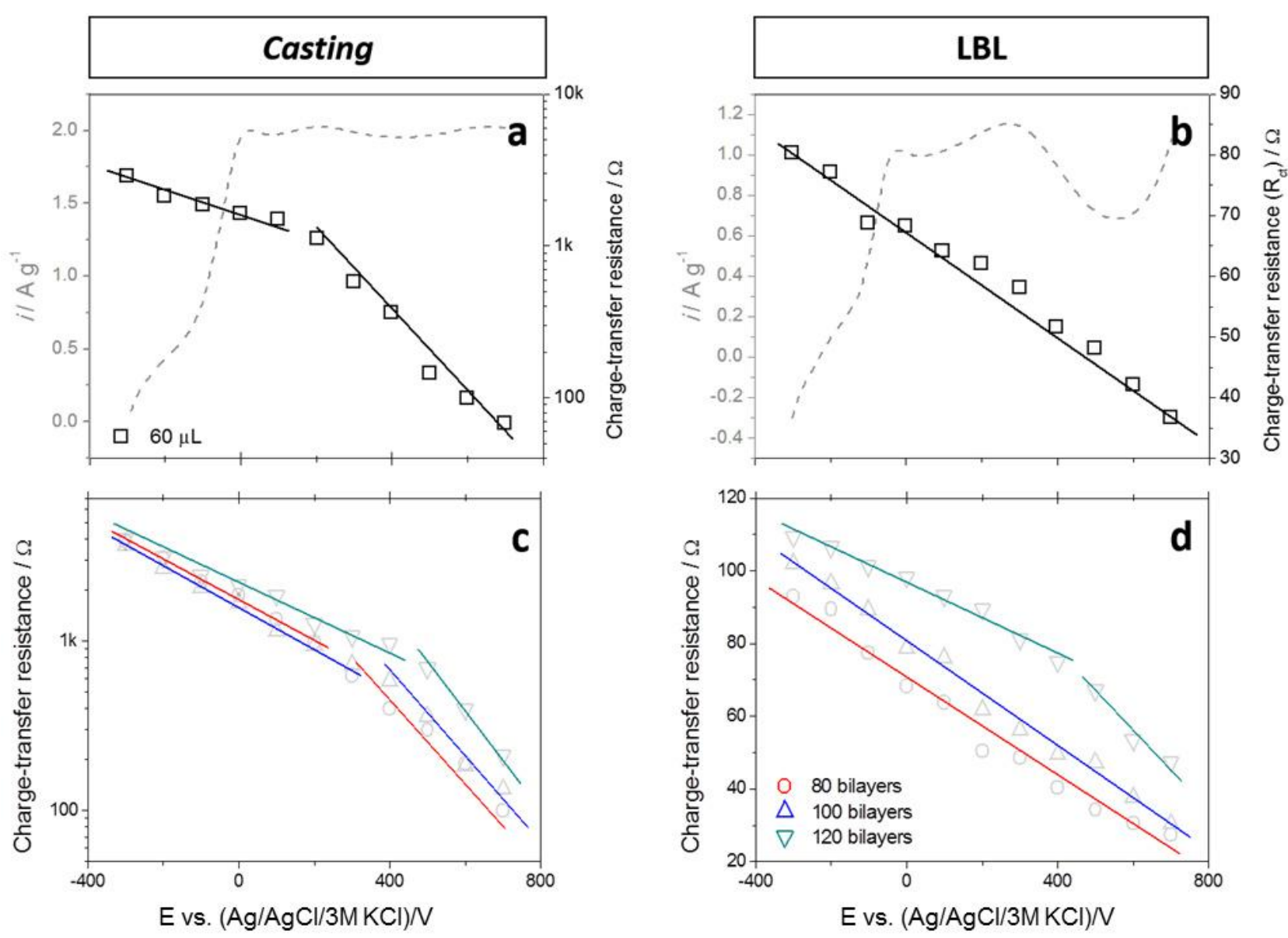

Figure 5: Dependence of $R_{\mathrm{ct}}$ on the applied potential for (a) $60 \mu \mathrm{L}$ cast polymer films and (b) 60 bilayers LBL films. The dashed line corresponds to the anodic sweep of the corresponding cyclic voltammogram, shown in Figure 3. The dependence on appied potential of $R_{\mathrm{ct}}$ of all the films prepared by polymer casting and LBL assembly are shown in (c) and (d), respectively.

EIS data can also be used to determine semi-quantitatively the degree of swelling of the films during the oxidation process. The uncompensated electrolyte resistance, $R_{\text {sol }}$ is directly proportional to the resistivity of the electrolyte $(\rho)$ and the separation between the tip of the reference and the working electrode $(L)$, and inversely proportional to the cross sectional area $(A$, $R_{\text {sol }}=\rho L / A$ ). $\rho$ and $A$ are constant, so any change in $R_{\text {sol }}$ must be due to changes in $L$. Similarly, $R_{\text {pore }}$ must depend on the shape of the pore, shorter and wider pores lead to lower $R_{\text {pore, }}$ although a lower availability of ions also cause this effect. In this sense, $R_{\text {sol }}$ and $R_{\text {pore }}$ here will be used as an indication of pore's form and volume change undergone by the film during the oxidation process. Since both $\mathrm{R}_{\text {sol }}$ and $\mathrm{R}_{\text {pore }}$ have different initial values for each tested film, it was decided to standardize and compare based on percentage of change. For this, $\mathrm{R}_{\text {pore }}$ starts at $0 \%$, indicating no 
deformation. With the oxidation process occurs this percentage rises, being interpreted as deformation percentage. Likewise, the $\mathrm{R}_{\text {sol }}$ starts at $0 \%$ and increases as the film swells. The increase in thickness due to polymer oxidation is already described in literature[62-64]. The change of this resistance are presented in Figure 6. In general, the films casting swell about two times $\left(\mathrm{R}_{\mathrm{pol}}\right.$ increase from 35 to $42 \%$ for different samples) more than the LBL film (from 13 to $21 \%$ ), and this fact is in agreement with the theoretical studies which will be discussed below.
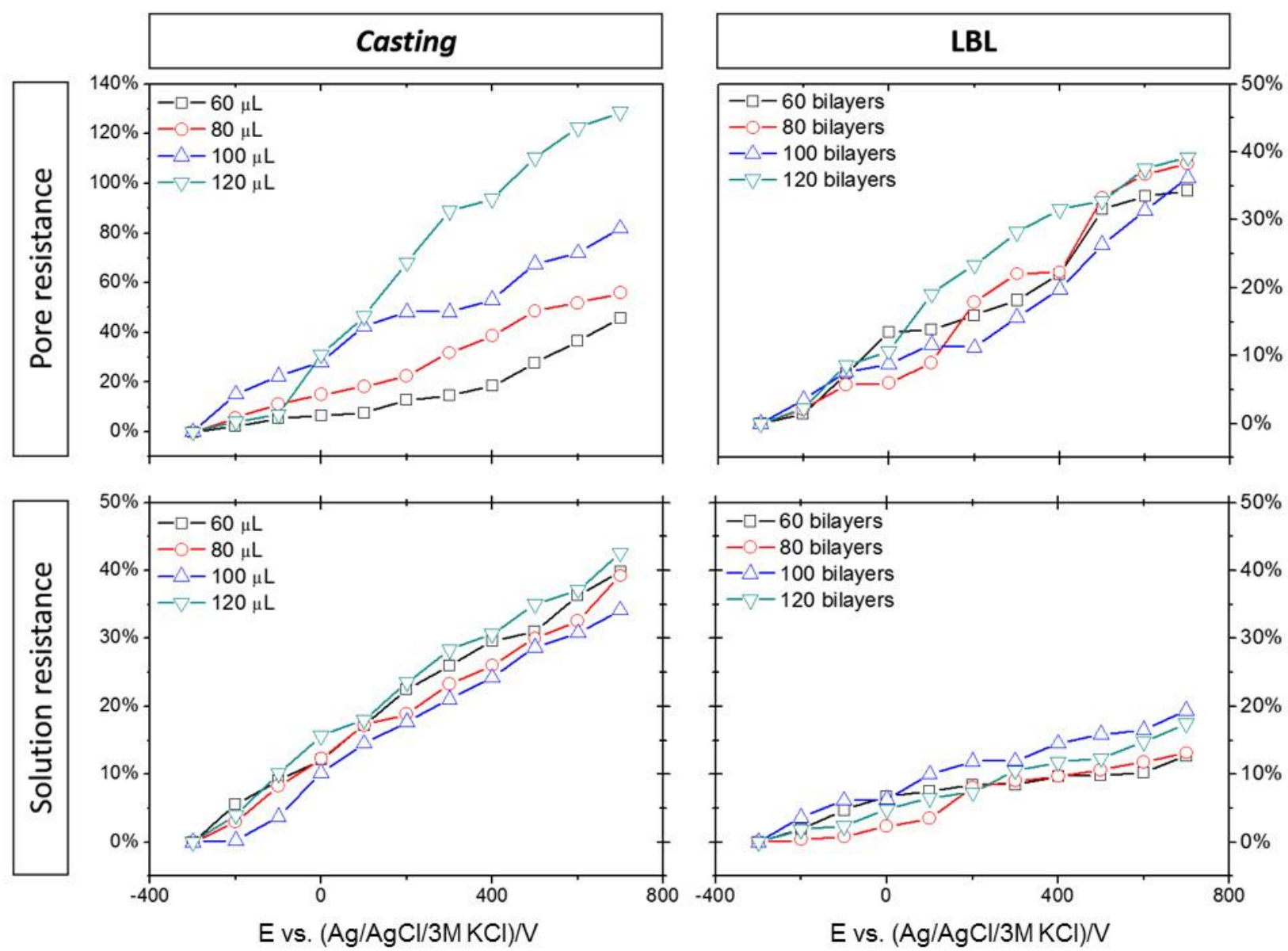

Figure 6: Relative changes of $R_{\text {pore }}$ (top) and $R_{\mathrm{ct}}$ (bottom) with applied potential for polymer cast films (left) and LBL films (right).

\subsection{Computational modelling of the redox behaviour of POMA films}

Using the impedance parameters it was possible to propose changes in film morphology of both materials, which are consistent with previous work involving this type of observation[37,63,65]. Thus, from a theoretical point of view, we use DFT calculations to describe, at a molecular level, those changes observed from electrochemical experiments and thus understand the mechanism of self-doping.

The essential difference between LBL and cast films lies in the counter ions balancing the positive charges generated upon oxidation (the PTAA layer in the case of $\mathrm{LBL}$ films, $\mathrm{ClO}_{4}{ }^{-}$in the 
case of cast polymer films) and their availability for the intercalation process. An adequate

description of PTAA and $\mathrm{ClO}_{4}{ }^{-}$within the computational model is, hence, necessary. $\mathrm{ClO}_{4}{ }^{-}$was first optimized separately, using the PM7 semi-empirical method, and then using DFT. The optimized structure was preserved during the computation of its interaction with POMA oligomers. The interaction of perchlorate with neutral POMA provokes changes in the intramolecular angles, as well as in some bond distances, as expected, because perchlorate promotes electronic repulsion of the close atoms. $\mathrm{C}-\mathrm{N}$ bonds are slightly elongated (from 1.46 to $1.47 \AA$ ), and the $\mathrm{C}-\mathrm{N}-\mathrm{C}$ bond angle increases by $1.30^{\circ}$. Even though these changes are not very large, it is important to bear in mind that they could represents an appreciable overall change, once propagated along the polymeric chain. Moreover, these changes are more pronounced for oxidized POMA, leading to an important structural reshaping. In the presence of perchlorate, the oxidation causes an increase in the C-N-C bond angle and a decrease of $\mathrm{C}-\mathrm{N}$ bond length of $15.8 \%$ and $6.12 \%$, respectively. Oxidation also provokes an increase of the dihedral angle of $38.6 \%$ in the presence of $\mathrm{ClO}_{4}{ }^{-}$, as opposed to $32.8 \%$ in its absence. This suggests that the presence of perchlorate leads to an increase in the twisting of the monomeric units, leading to a slight chain shortening. Macroscopically, such shortening is associated with a change in volume that, as described in different papers[62,66,67] and in Figure 6 , could be responsible for the polymer degradation when submitted to electrochemical cycles.

The PTAA chain was simulated following previous DFT work, in which the poly(3hexylthiophene) was simulated[35]. In the present case, we describe only four monomeric units applying PBC to simulate long polymer chains. PTAA displays a helicoid structure, each monomeric unit twisting with respect to the previous one by approximately $60^{\circ}$. Mulliken charge analysis shows charge accumulation at the $-\mathrm{COO}^{-}$residue, with $-0.599,-0.822$ and -0.831 at $\mathrm{C},=\mathrm{O}$ and $-\mathrm{O}^{-}$, respectively. In addition, the $-\mathrm{COO}^{-}$group has resonance as demonstrated by $\mathrm{BO} 1.47$ for $\mathrm{C}-\mathrm{O}$ bonds.

In the POMA/PTAA case, the $\mathrm{C}-\mathrm{N}$ bonds present smaller values which could be related to the $\pi-\pi$ interaction between the two chains. There is a decrease in the POMA dihedral angles, from $37.2^{\circ}$ to $28.4^{\circ}$, meaning that the POMA monomeric units are less twisted due to their interaction with PTAA chain. For the POMA/PTAA oxidized supermolecules, there is also a decrease in these parameters compared to pure POMA one. As consequence, in the LBL film, POMA does not swell too much due to the decrease in the torsion of the aromatic rings. It is also observed a chain shortening of $4.23 \%$ and $9.22 \%$ bond angle decrease. Table 2 summarizes all the calculated changes (The absolute values are present in Table S1 in the Supplementary Information). 
Table 2: Changes in important structural parameters upon POMA oxidation as obtained by DFT calculations.

\section{Pure POMA POMA + $\mathrm{ClO}_{4}^{-} \quad$ POMA/PTAA}

\begin{tabular}{c|ccc} 
C-N bond lengh / \% & -6.85 & -6.12 & -4.23 \\
$\boldsymbol{C}-\boldsymbol{N}-\boldsymbol{C}$ angle / \% & 14.4 & 15.8 & 9.22 \\
Dihedral angle / \% & 32.8 & 38.6 & 20.8
\end{tabular}

The interaction between POMA and each molecule revealed a very significant result. For reduced POMA/PTAA, a total energy reduction was observed, which is1.96 Eh (53.22 $\left.\mathrm{eV} \mathrm{mol}^{-1}\right)$ compared to pure POMA. On the other hand, only a small decrease in energy, 0.0449 Eh $(0.127$ $\mathrm{eV} \mathrm{mol}^{-1}$ ), is observed for reduced POMA+ClO${ }_{4}^{-}$. This is result means that POMA/PTAA is much more stable than POMA $+\mathrm{ClO}_{4}{ }^{-}$supermolecules.

We have also calculated the interaction of two POMA chains, reduced and positively charged. As expected, in the case of oxidation of cast polymer chains, the layers tend to separate due to electrostatic repulsion (Figure 7a), which is somewhat minimized by intercalation of counterions. On the contrary, this separation is not observed in the case of the interaction of POMA and PTAA in the LBL structure (Figure $7 b$ ). This is also an expected result because, the protonation/deprotonation of the $\mathrm{COOH}$ residue in the PTAA acts as charge compensating, and thereby charge repulsion is avoided. In order to test this hypothesis, an additional computational experiment was carried out to calculate the equilibrium distance between two chains. In the case of two POMA chains, equilibrium distances are $3.60 \AA$ for reduced and $3.85 \AA$ for positively charged molecules, i.e., the equilibrium distance increases by $6.9 \%$ upon oxidation. On the other hand, in the case of POMA/PTAA a $1.2 \%$ decrease from 3.42 to $3.38 \AA$ in the equilibrium interchain distance was found, in agreement with the proposition that the volume change is smaller in the case of LBL films. Energy curve plots are presented in Figure S4 of the Supplementary Information. 
Casting
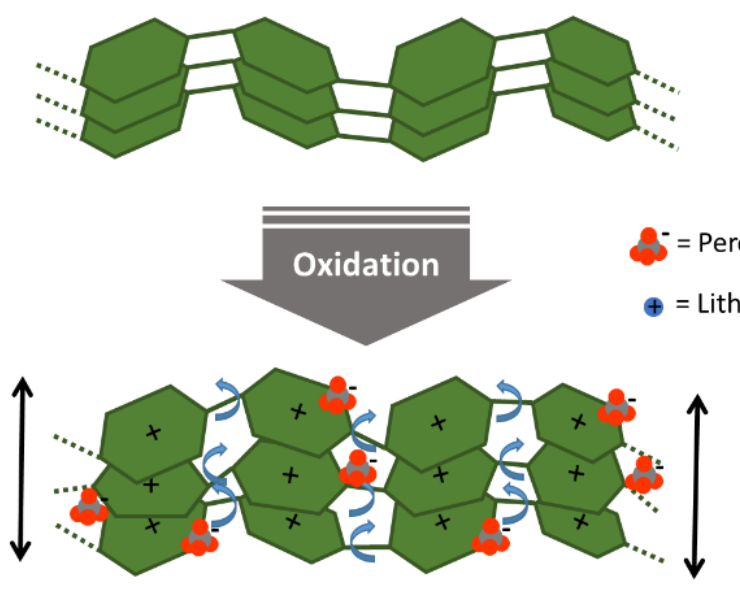

LBL

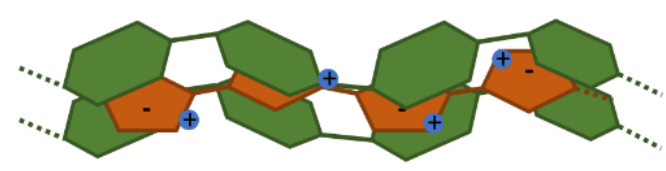

\section{Oxidation}

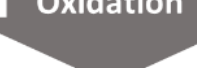

Figure 7: Graphical representation of polymer chain behaviour during the oxidation process in the presence of the anion and the polyanion.

Looking at both series of experiments it is possible to correlate the magnitude of film swelling due to inherent redox process of conducting polymers with its durability. Cast films depend on ion intercalation from the solution for charge neutralisation, which requires a mass transport step, leading to low response times and short life-time due to the concomitant structure changes. On the contrary, LBL films have an in-built charge-compensation system, which suppresses the mass transport step at least partially, and reduces the extent of conformational change associated to the oxidation of the polymer chains, decreasing the response time and increasing the material's lifetime.

\section{CONLUSIONS}

The results shown that layer-by-layer POMA/PTAA films have improved electrochemical properties such as capacitance values. It has been shown that the positively and negatively charged layers, i.e., POMA and PTAA, respectively, presents conformational changes and electrochemical behaviour compared to cast POMA samples. Furthermore, the computational experiments shown an additional decrease in the energy minimization compared to individual polymer layers. From a thermodynamic point of view, this is an important issue to explain the enhance in the film stability. Besides, in the LBL films, the twisting of the polymer chain due to the oxidation is $12 \%$ less while the chain shortening is 5\% compared with the casting ones. This theoretical result is supported by the EIS data, which showed a decrease in the swelling of the LBL films of $50 \%$ compared to casting ones. Finally, all these results lead us to proposed that the PTAA acts beyond a chargecompensator layer, working as a backbone structure that mechanically stabilize the POMA layer, leading to an increase in the durability, and improving the electrochemical properties. 


\section{ACKNOWLEDGMENTS}

The support of this research by FAPESP (2011/10897-2, 2013/07296-2), CsF-PVE (99999.007708/2015-07), CAPES and CNPq is gratefully acknowledged. We also thank the University of Aberdeen for providing computational time on Maxwell.

\section{REFERENCES}

[1] M.E. Abdelhamid, A.P. O’Mullane, G. a. Snook, Storing energy in plastics: a review on conducting polymers and their role in electrochemical energy storage, RSC Adv. 5 (2015) 11611-11626. doi:10.1039/C4RA15947K.

[2] A.M. Bryan, L.M. Santino, Y. Lu, S. Acharya, J.M. D'Arcy, Conducting Polymers for Pseudocapacitive Energy Storage, Chem. Mater. (2016) acs.chemmater.6b01762. doi:10.1021/acs.chemmater.6b01762.

[3] G. Inzelt, Rise and rise of conducting polymers, J. Solid State Electrochem. 15 (2011) 17111718. doi:10.1007/s10008-011-1338-3.

[4] R. Ramya, R. Sivasubramanian, M.V. Sangaranarayanan, Conducting polymers-based electrochemical supercapacitors-Progress and prospects, Electrochim. Acta. 101 (2013) 109-129. doi:10.1016/j.electacta.2012.09.116.

[5] E. Frackowiak, V. Khomenko, K. Jurewicz, K. Lota, F. Béguin, Supercapacitors based on conducting polymers/nanotubes composites, J. Power Sources. 153 (2006) 413-418. doi:10.1016/j.jpowsour.2005.05.030.

[6] X. Zhao, B.M. Sanchez, P.J. Dobson, P.S. Grant, The role of nanomaterials in redox-based supercapacitors for next generation energy storage devices, Nanoscale. 3 (2011) 839-855. doi:10.1039/c0nr00594k.

[7] M. Mastragostino, C. Arbizzani, F. Soavi, Polymer-based supercapacitors, J. Power Sources. 97-98 (2001) 812-815. doi:10.1016/S0378-7753(01)00613-9.

[8] M. Mastragostino, C. Arbizzani, F. Soavi, Conducting polymers as electrode materials in supercapacitors, 148 (2002) 493-498.

[9] X. Zhang, Z. Lin, B. Chen, W. Zhang, S. Sharma, W. Gu, et al., Solid-state flexible polyaniline/silver cellulose nanofibrils aerogel supercapacitors, J. Power Sources. 246 (2014) 
283-289. doi:10.1016/j.jpowsour.2013.07.080.

[10] G.A. Snook, P. Kao, A.S. Best, Conducting-polymer-based supercapacitor devices and electrodes, J. Power Sources. 196 (2011) 1-12. doi:10.1016/j.jpowsour.2010.06.084.

[11] G.A. Snook, P. Kao, A.S. Best, Conducting-polymer-based supercapacitor devices and electrodes, J. Power Sources. 196 (2011) 1-12. doi:10.1016/j.jpowsour.2010.06.084.

[12] B. Scrosati, Conducting Polymers and Their Applications, Mater. Sci. Forum. 42 (1989) 207-220. doi:10.4028/www.scientific.net/MSF.42.207.

[13] T.F. Otero, Soft, wet, and reactive polymers. Sensing artificial muscles and conformational energy, J. Mater. Chem. 19 (2009) 681-689. doi:10.1039/B809485C.

[14] A. Malinauskas, Self-doped polyanilines, J. Power Sources. 126 (2004) 214-220. doi:10.1016/j.jpowsour.2003.08.008.

[15] F. Trivinho-Strixino, E.C. Pereira, S. V Mello, O.N. Oliveira, Self-doping effect in poly(omethoxyaniline)/poly(3-thiopheneacetic acid) layer-by-layer films., Langmuir. 20 (2004) 3740-5. http://www.ncbi.nlm.nih.gov/pubmed/15875409.

[16] W.A. Christinelli, R. Gonçalves, E.C. Pereira, A new generation of electrochemical supercapacitors based on layer-by-layer polymer films, J. Power Sources. 303 (2016) 73-80. doi:10.1016/j.jpowsour.2015.10.077.

[17] W.A. Christinelli, R. Gonçalves, E.C. Pereira, Optimization of electrochemical capacitor stability of poly(o-methoxyaniline)-poly(3-thiophene acetic acid) self-assembled films, Electrochim. Acta. (2016). doi:10.1016/j.electacta.2016.02.187.

[18] W.A. Christinelli, A.B. Trench, E.C. Pereira, Electrochromic properties of poly(omethoxyaniline)-poly(3-thiophene acetic acid) layer by layer films, Sol. Energy Mater. Sol. Cells. 157 (2016) 703-708. doi:10.1016/j.solmat.2016.07.035.

[19] G. Garcia-Belmonte, A. Munar, E.M. Barea, J. Bisquert, I. Ugarte, R. Pacios, Charge carrier mobility and lifetime of organic bulk heterojunctions analyzed by impedance spectroscopy, Org. Electron. 9 (2008) 847-851. doi:10.1016/j.orgel.2008.06.007.

[20] G. Garcia-Belmonte, J. Bisquert, E.C. Pereira, F. Fabregat-Santiago, Anomalous transport on polymeric porous film electrodes in the dopant-induced insulator-to-conductor transition analyzed by electrochemical impedance, Appl. Phys. Lett. 78 (2001) 1885. doi:10.1063/1.1354671.

[21] J. Smith, P. Cox, N.M. Ratcliffe, S. Campbell, Conducting polymers as coatings: Electrochemical and density functional theory investigations of the polymerisation, Trans. 
Inst. Met. Finish. (2002). http://eprints.uwe.ac.uk/19765/ (accessed July 17, 2015).

[22] N. Maouche, B. Nessark, Cyclic Voltammetry and Impedance Spectroscopy Behavior Studies of Polyterthiophene Modified Electrode, Int. J. Electrochem. 2011 (2011) 1-5. doi:10.4061/2011/670513.

[23] W.-C. Chen, T.-C. Wen, C.-C. Hu, a Gopalan, Identification of inductive behavior for polyaniline via electrochemical impedance spectroscopy, Electrochim. Acta. 47 (2002) 1305-1315. doi:10.1016/S0013-4686(01)00849-0.

[24] J.E.B. Randles, Kinetics of rapid electrode reactions, Discuss. Faraday Soc. 1 (1947) 11. doi:10.1039/df9470100011.

[25] G. Paasch, K. Micka, P. Gersdorf, Theory of the electrochemical impedance of macrohomogeneous porous electrodes, Electrochim. Acta. 38 (1993) 2653-2662. doi:10.1016/0013-4686(93)85083-B.

[26] G. Paasch, Complete electrochemical transmission line model for conducting polymers, Synth. Met. 119 (2001) 233-234. doi:10.1016/S0379-6779(00)00865-1.

[27] J.P. Meyers, M. Doyle, R.M. Darling, J. Newman, The Impedance Response of a Porous Electrode Composed of Intercalation Particles, J. Electrochem. Soc. 147 (2000) 2930. doi:10.1149/1.1393627.

[28] M. Schlesinger, ed., Modern Aspects of Electrochemistry, Number 43, Springer New York, New York, NY, 2009. doi:10.1007/978-0-387-49582-8.

[29] J. Bisquert, A. Compte, Theory of the electrochemical impedance of anomalous diffusion, J. Electroanal. Chem. 499 (2001) 112-120. doi:10.1016/S0022-0728(00)00497-6.

[30] I. Levine, Quantum Chemistry, 7 edition, Prentice Hall, 2013.

[31] J.P. Perdew, K.A. Jackson, M.R. Pederson, D.J. Singh, C. Fiolhais, Atoms, molecules, solids, and surfaces: Applications of the generalized gradient approximation for exchange and correlation, Phys. Rev. B. 46 (1992) 6671-6687. doi:10.1103/PhysRevB.46.6671.

[32] A. Ehsani, F. Babaei, H. Mostaanzadeh, Electrochemical and Optical Investigation of Conductive Polymer and MWCNT Nanocomposite Film, J. Braz. Chem. Soc. 26 (2014) 331-337. doi:10.5935/0103-5053.20140284.

[33] J.A. Schmidt, R.E. Koehn, T.M. Pappenfus, J.D. Alia, PBC-DFT : An Efficient Method to Calculate Energy Band Gaps of Conducting Polymers used in Solar Cells, (2010). http://conservancy.umn.edu/handle/11299/101775 (accessed July 17, 2015). 
[34] S. Yang, P. Olishevski, M. Kertesz, Bandgap calculations for conjugated polymers, Synth. Met. 141 (2004) 171-177. doi:10.1016/j.synthmet.2003.08.019.

[35] R. Gonçalves, F.C. Moraes, E.C. Pereira, Reduced graphene oxide enhancing the photoelectrochemical properties of poly(3-hexylthiophene), Carbon N. Y. (2016). doi:10.1016/j.carbon.2016.07.041.

[36] W.A. Christinelli, R. Gonçalves, E.C. Pereira, A new generation of electrochemical supercapacitors based on layer-by-layer polymer films, J. Power Sources. 303 (2016) 73-80. doi:10.1016/j.jpowsour.2015.10.077.

[37] W.A. Christinelli, R. Gonçalves, E.C. Pereira, Optimization of electrochemical capacitor stability of poly(o-methoxyaniline)-poly(3-thiophene acetic acid) self-assembled films, Electrochim. Acta. 196 (2016) 741-748. doi:10.1016/j.electacta.2016.02.187.

[38] F. Trivinho-Strixino, E.C. Pereira, L.R.C. Lopes, Desenvolvimento de um sistema automatizado para a fabricação de filmes automontados, Quim. Nova. 27 (2004) 661-663. doi:10.1590/S0100-40422004000400022.

[39] J.J.P. Stewart, Optimization of parameters for semiempirical methods VI: more modifications to the NDDO approximations and re-optimization of parameters, J. Mol. Model. 19 (2013) 1-32. doi:10.1007/s00894-012-1667-x.

[40] S.C.C. James Stewart, MOPAC2016, (2016). http://openmopac.net/.

[41] J.D.C. Maia, G.A. Urquiza Carvalho, C.P. Mangueira, S.R. Santana, L.A.F. Cabral, G.B. Rocha, GPU Linear Algebra Libraries and GPGPU Programming for Accelerating MOPAC Semiempirical Quantum Chemistry Calculations, J. Chem. Theory Comput. 8 (2012) 30723081. doi:10.1021/ct3004645.

[42] J. VandeVondele, J. Hutter, Gaussian basis sets for accurate calculations on molecular systems in gas and condensed phases, J. Chem. Phys. 127 (2007) 114105. doi:10.1063/1.2770708.

[43] A.D. Becke, Density-functional exchange-energy approximation with correct asymptotic behavior, Phys. Rev. A. 38 (1988) 3098-3100. doi:10.1103/PhysRevA.38.3098.

[44] J. Hutter, M. Iannuzzi, F. Schiffmann, J. VandeVondele, cp2k: atomistic simulations of condensed matter systems, Wiley Interdiscip. Rev. Comput. Mol. Sci. 4 (2014) 15-25. doi:10.1002/wcms.1159.

[45] Maxwell High Performance Computing Cluster, (n.d.). www.abdn.ac.uk/staffnet/research/research-computing. 
[46] M.D. Hanwell, D.E. Curtis, D.C. Lonie, T. Vandermeersch, E. Zurek, G.R. Hutchison, Avogadro: an advanced semantic chemical editor, visualization, and analysis platform, J. Cheminform. 4 (2012) 17. doi:10.1186/1758-2946-4-17.

[47] T. Laino, F. Mohamed, A. Laio, M. Parrinello, An Efficient Linear-Scaling Electrostatic Coupling for Treating Periodic Boundary Conditions in QM/MM Simulations, J. Chem. Theory Comput. 2 (2006) 1370-1378. doi:10.1021/ct6001169.

[48] M. Del Ben, M. Schönherr, J. Hutter, J. VandeVondele, Bulk Liquid Water at Ambient Temperature and Pressure from MP2 Theory, J. Phys. Chem. Lett. 4 (2013) 3753-3759. doi:10.1021/jz401931f.

[49] G. Ćirić-Marjanović, Recent advances in polyaniline research: Polymerization mechanisms, structural aspects, properties and applications, Synth. Met. 177 (2013) 1-47. doi:10.1016/j.synthmet.2013.06.004.

[50] R.S. Mulliken, Electronic Population Analysis on LCAO[Single Bond]MO Molecular Wave Functions. I, J. Chem. Phys. 23 (1955) 1833. doi:10.1063/1.1740588.

[51] S. Sinnecker, A. Rajendran, A. Klamt, M. Diedenhofen, F. Neese, Calculation of solvent shifts on electronic g-tensors with the conductor-like screening model (COSMO) and its selfconsistent generalization to real solvents (direct COSMO-RS)., J. Phys. Chem. A. 110 (2006) 2235-45. doi:10.1021/jp056016z.

[52] A.F. Diaz, J.A. Logan, Electroactive polyaniline films, J. Electroanal. Chem. Interfacial Electrochem. 111 (1980) 111-114. doi:10.1016/S0022-0728(80)80081-7.

[53] A.K. Mishra, DFT study of structural, vibrational and electronic properties of polyaniline pernigraniline model compounds, J. Comput. Sci. 10 (2015) 195-208. doi:10.1016/j.jocs.2015.02.003.

[54] A.K. Mishra, P. Tandon, A Comparative Ab Initio and DFT Study of Polyaniline Leucoemeraldine Base and Its Oligomers, J. Phys. Chem. B. 113 (2009) 14629-14639. doi:10.1021/jp906799m.

[55] X.P. Chen, J.K. Jiang, Q.H. Liang, N. Yang, H.Y. Ye, M. Cai, et al., First-principles study of the effect of functional groups on polyaniline backbone, Sci. Rep. 5 (2015) 16907. doi:10.1038/srep16907.

[56] W.-S. Huang, B.D. Humphrey, A.G. MacDiarmid, Polyaniline, a novel conducting polymer. Morphology and chemistry of its oxidation and reduction in aqueous electrolytes, J. Chem. Soc. Faraday Trans. 1 Phys. Chem. Condens. Phases. 82 (1986) 2385. 
doi:10.1039/f19868202385.

[57] E.M. Genies, C. Tsintavis, Redox mechanism and electrochemical behaviour or polyaniline deposits, J. Electroanal. Chem. Interfacial Electrochem. 195 (1985) 109-128. doi:10.1016/0022-0728(85)80009-7.

[58] E.M. Genies, M. Lapkowski, Polyaniline films. Electrochemical redox mechanisms, Synth. Met. 24 (1988) 61-68. doi:10.1016/0379-6779(88)90595-4.

[59] A.G. Macdiarmid, J.C. Chiang, A.F. Richter, A.J. Epstein, Polyaniline: a new concept in conducting polymers, Synth. Met. 18 (1987) 285-290. doi:10.1016/0379-6779(87)90893-9.

[60] K. Darowicki, J. Kawula, Impedance characterization of the process of polyaniline first redox transformation after aniline electropolymerization, Electrochim. Acta. 49 (2004) 4829-4839. doi:10.1016/j.electacta.2004.05.035.

[61] E.I. Santiago, E.C. Pereira, L.O.S. Bulhões, Characterization of the redox processes in polyaniline using capacitance-potential curves, Synth. Met. 98 (1998) 87-93. doi:10.1016/S0379-6779(98)00149-0.

[62] T.F. Otero, Biomimetic Conducting Polymers: Synthesis, Materials, Properties, Functions, and Devices, Polym. Rev. 53 (2013) 311-351. doi:10.1080/15583724.2013.805772.

[63] R. Gonçalves, A.A. Correa, R. Pereira, E.C. Pereira, Investigation of the electrochemical aging of poly(3-hexiltiophene) using impedance spectroscopy, Electrochim. Acta. 190 (2016) 329-336. doi:10.1016/j.electacta.2015.12.198.

[64] X. Jiang, Y. Harima, R. Patil, A transport study on as-grown and cast films of electrogenerated poly(3-hexylthiophene), Mater. Lett. 61 (2007) 4687-4689. doi:10.1016/j.matlet.2007.03.010.

[65] A.A. Correa, R. Gonçalves, R. Pereira, E.C. Pereira, The electropolymerization of several poly(3-methylthiophene) films in the same used solution and its consequence in their properties, J. Appl. Polym. Sci. (2016). doi:10.1002/app.44368.

[66] W. Zheng, J.M. Razal, G.M. Spinks, V.T. Truong, P.G. Whitten, G.G. Wallace, The role of unbound oligomers in the nucleation and growth of electrodeposited polypyrrole and method for preparing high strength, high conductivity films, Langmuir. 28 (2012) 10891-10897. doi:10.1021/la301701g.

[67] Y. Harima, X. Jiang, R. Patil, K. Komaguchi, H. Mizota, Influence of film structure on mobilities of charge carriers in conducting polymers, Electrochim. Acta. 52 (2007) 80888095. doi:10.1016/j.electacta.2007.07.007. 


\section{FIGURE CAPTIONS}

Figure 1: Graphical representation of the two-braches (top) and the one-branch (bottom) transmission-lines models used to analyse the EIS data.

Figure 2: Cyclic voltammograms for cast POMA (left) and LBL POMA/PTAA films (right) in 0.1 mol L-1 LiClO4 in actenitrile at $20 \mathrm{mV} \mathrm{s}-1$. (a) 60 (b) 80, (c) 100 and (d) $120 \mu \mathrm{L}$; (e) 60 (f) 80, (g) 100 and (h) 120 bilayers; performed in LiClO4 $0.1 \mathrm{~mol} \mathrm{L-1/ACN}$ at $20 \mathrm{mV} \mathrm{s}-1$.

Figure 3: Bode plots for $80 \mathrm{uL}$ POMA cast films (left) and 60 bilayers LBL films (right) in 0.1 mol $\mathrm{L}-1 \mathrm{LiClO} 4$ in acetonitrile at $-0.30 \mathrm{~V}$ (black squares) and $0.70 \mathrm{~V}$ (blue circles). $\Delta V=10 \mathrm{mV}$.

Figure 4: Double-layer capacitance as function of applied potential for casting and LBL films.

Figure 5: Dependence of Rct on the applied potential for (a) $60 \mu \mathrm{L}$ cast polymer films and (b) 60 bilayers LBL films. The dashed line corresponds to the anodic sweep of the corresponding cyclic voltammogram, shown in Figure 3. The dependence on appied potential of Rct of all the films prepared by polymer casting and LBL assembly are shown in (c) and (d), respectively.

Figure 6: Relative changes of Rpore (top) and Rct (bottom) with applied potential for polymer cast films (left) and LBL films (right).

Figure 7: Graphical representation of polymer chain behaviour during the oxidation process in the presence of the anion and the polyanion. 


\section{TABLE CAPTIONS}

Table 1: Some structural parameters from the DFT calculations for POMA in reduced and oxidized states.

Table 1: Some structural parameters from the DFT calculations for POMA in reduced and oxidized states.Table 2: Changes in important structural parameters upon POMA oxidation as obtained by DFT calculations. 
Table 1: Some structural parameters from the DFT calculations for POMA in reduced and oxidized states.

\begin{tabular}{c|cc} 
& $\begin{array}{c}\text { Neutral POMA (reduced } \\
\text { form) }\end{array}$ & $\begin{array}{c}\text { Positively charged POMA } \\
\text { (oxidised form) }\end{array}$ \\
\hline Aromatic C-C bond length / $\AA$ & 1.40 & $1.51 / 1.37$ \\
Aromatic C-C bond order & 1.49 & $1.12 / 1.97$ \\
C-N bond length / $\AA$ & 1.46 & 1.36 \\
C-N bond order & 0.988 & 1.51 \\
C-N-C angle $^{\circ}$ & 119.6 & 136.4 \\
Rings dihedral angle $^{\circ}$ & 37.2 & 49.4 \\
N Mulliken charge & -0.618 & +0.987 \\
O Mulliken charge & -0.563 & -0.568
\end{tabular}


Table 2: Changes in important structural parameters upon POMA oxidation as obtained by DFT calculations.

\begin{tabular}{|c|c|c|c|}
\hline & Pure POMA & $\mathrm{POMA}+\mathrm{ClO}_{4}^{-}$ & РОМА/РТАА \\
\hline$C-N$ bond lengh /\% & -6.85 & -6.12 & -4.23 \\
\hline $\mathrm{C}-\mathrm{N}-\mathrm{C}$ angle $/ \%$ & 14.4 & 15.8 & 9.22 \\
\hline Dihedral angle / \% & 32.8 & 38.6 & 20.8 \\
\hline
\end{tabular}



Supplementary Materials
Click here to download Supplementary Materials: Supplementary_2016_11_01_Roger.docx

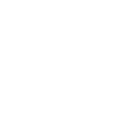

t

.

(1)

(1)

(1)

(1)

